\title{
Mapping and rehabilitation of brownfields in the city of Izhevsk
}

\author{
V. I. Sturman \\ Udmurt State University, Russian Federation, Russia
}

\begin{abstract}
The term "Brownfield" has no equivalent in the Russian language, but the problem arises in every industrial city. Geochemical mapping of Izhevsk municipal area was conducted in the 1990s. According to its results, high degrees of soil contamination were discovered within industrial zones and in nearby territories. There are several examples of rehabilitation projects in Izhevsk, and they show various remediation strategies.

One of the residential districts in Izhevsk was built on the site of a disused airport. The remainder of pesticides for agricultural aviation was not moved off. The cleanup project was completed later by excavation of contaminated soil.

A few years ago the building of a former ball bearing plant was reconstructed as an office center. Environmental investigation of the site revealed dangerous soil and groundwater contamination by lubricating oil. The cleanup was carried out by pumping oil through special bore-wells.

The most difficult situation is connected with metallurgical works. From the1760s till the 1980s metallurgical slag had been deposited on the cove of the Izhevsk pond. Slag increases alkalinity of the groundwater, and alkaline water, in its turn, lixiviates 6-valent chromium from slag into the pond. Since excavation is not possible in the mentioned case, our project is based on creating an artificial geochemical barrier for chromium ions deposition. Natural peat will be used as the main material for the geochemical barrier.
\end{abstract}

Keywords: geochemical mapping, remediation strategies, heavy metals, pesticides, lubricating oil. 


\section{Theory and technique}

The term "Brownfield" first came into use a couple of decades ago. It means abandoned contaminated land that used to serve non-agricultural purposes such as industry or mining [1].

Russian legislation requires developing companies to carry out environmental site investigations in all cases of civil or industrial projecting [2]. Rehabilitation is obligatory for the sites with significant soil contamination. Property owner as the interested party covers the expenses related to the site investigation. The polluter de jure covers rehabilitation expenses, but practically it is the interested owner who pays them, especially in case of brownfield sites with a long history.

The technique of geochemical mapping of urban territories was elaborated by the Institute of Mineralogy, Geochemistry and Crystallochemistry of Scattered Elements, Russian Academy of Sciences [3], and applied in many cities and towns of Russia.

The technique includes: surface soil (or snow) sampling; chemical analysis of the samples; calculation and mapping of contamination indexes; health and toxicological investigations. The mentioned index values are concentration indexes of the specific chemical elements $(\mathrm{Kc})$ and total contamination index $(\mathrm{Zc})$ :

$$
\mathrm{Kc}=\frac{\mathrm{Ci}}{\mathrm{Cn}}
$$

C - concentration of the specific element in the sample;

$\mathrm{Cn}$ - natural (background) concentration of the element in the same kind of solution outside of the contaminated areas.

$$
\mathrm{Zc}=\Sigma \mathrm{Kc}-(\mathrm{n}-1),
$$

$\mathrm{n}$ - number of terms in the Kc sum.

Grade of contamination has been considering permissible if $\mathrm{Zc}$ not more 16 , moderate dangerous if $\mathrm{Zc} 16-32$, dangerous if $\mathrm{Zc} 32-128$ and extremely dangerous if $\mathrm{Zc}$ more 128. The scale is based on toxicological investigations carried out by governmental environment institutions in the former USSR and can be compared to the soil pollution indexes developed by Sanka et.al. [4].

The number of samples can be rather seldom or a single in case of development project, but it can also rise up to hundreds and thousands in case of fully-fledged geochemical mapping. At first, the sample is analysed for the presence of heavy and toxic metals $(\mathrm{Pb}, \mathrm{Zn}, \mathrm{Cu}, \mathrm{Ni}, \mathrm{Co}, \mathrm{As}, \mathrm{Cd}, \mathrm{Hg})$. These elements indicate other types of contamination, so if their concentration is above average, additional investigations are carried out. Waterbody bottom deposits and snow can be additional objects of sampling. The results of geochemical mapping are usually presented by isoline maps of $\mathrm{Kc}$ and $\mathrm{Zc}$. The grades of contamination are classified using a level scale of Zc.

The main sources of soil contamination are: air pollution through atmospheric sedimentation (causes so-called aerogenic geochemical anomalies), water 
deposition (hydrogenic anomalies), dumping of waste (waste formed anomalies), application of fertilisers and pesticides (agrogenic anomalies) [3]. Aerogenic anomalies usually affect surface soil layers and have diffuse outlines. Other kinds of anomalies are characterized by more specific location and chemical composition.

The type of anomaly is determined by analysis of its chemical composition, location and outline as compared to the location and waste composition of pollution sources in the neighbourhood. Multi-layer soil sampling through excavation and drilling is a way to elucidate the origin in the most difficult cases.

\section{The mapping of soils contamination in Izhevsk}

Izhevsk (645000 inhabitants) is located in the eastern part of the Russian plain, $300 \mathrm{~km}$ west from the Ural mountains, in the zone of mixed forests, outside of Quaternary glacial region. The city was founded in 1760 at the same time as the metallurgical works and the dam on the river Izh, right tributary of the Kama. Podzols are the dominant soil type in the region.

Mapping of soil contamination in Izhevsk is based on the chemical analysis of more than 3000 samples. Background concentrations of chemical elements were determined from analogous soils and deposits in the countryside areas 80-100 $\mathrm{km}$ west and north-east from Izhevsk. The results are presented in figure 1.

Corresponding maps for $\mathrm{Pb}, \mathrm{Zn}, \mathrm{Cu}, \mathrm{Ni}, \mathrm{Mn}, \mathrm{Cr}, \mathrm{V}$ were also compiled. The results of mapping show dependencies between contamination level and land use, relief, landscapes. The most contaminated areas of Izhevsk are:

- the industrial zone in the city centre (metallurgical and engineering works);

- the storage of metallurgical slag;

- the industrial zone constructed on made ground in the floodplain of the river Izh;

- the industrial zone in the north-eastern part of the city (non-ferrous foundry and car plant).

Less serious soil contamination takes place in the downtown (large aerogenic anomaly), within and near minor industrial areas and on the floodplains of small rivers (hydrogenic anomalies). There are totally 20 large anomalies, connected with industrial zones, and about 200 small anomalies near minor industrial properties, main roads and in the residential area.

\section{Relief and landscape influence on soil contamination}

Landscape features can affect soil contamination both directly, by redistribution of air-carried industrial dust, and indirectly, by imposing land use strategy. Maps of soil contamination were compared with large-scale maps of landscapes in order to investigate these influences. Table 1 shows average concentration of elements and total contamination indexes Zc among various landforms as well as in Quaternary and Permian deposits. The distribution of contamination indexes reflects the prior development of floodplains and gentle slopes. $80 \%$ of the areas with dangerous contamination $(\mathrm{Zc}>32)$ are located in the floodplains and gentle 


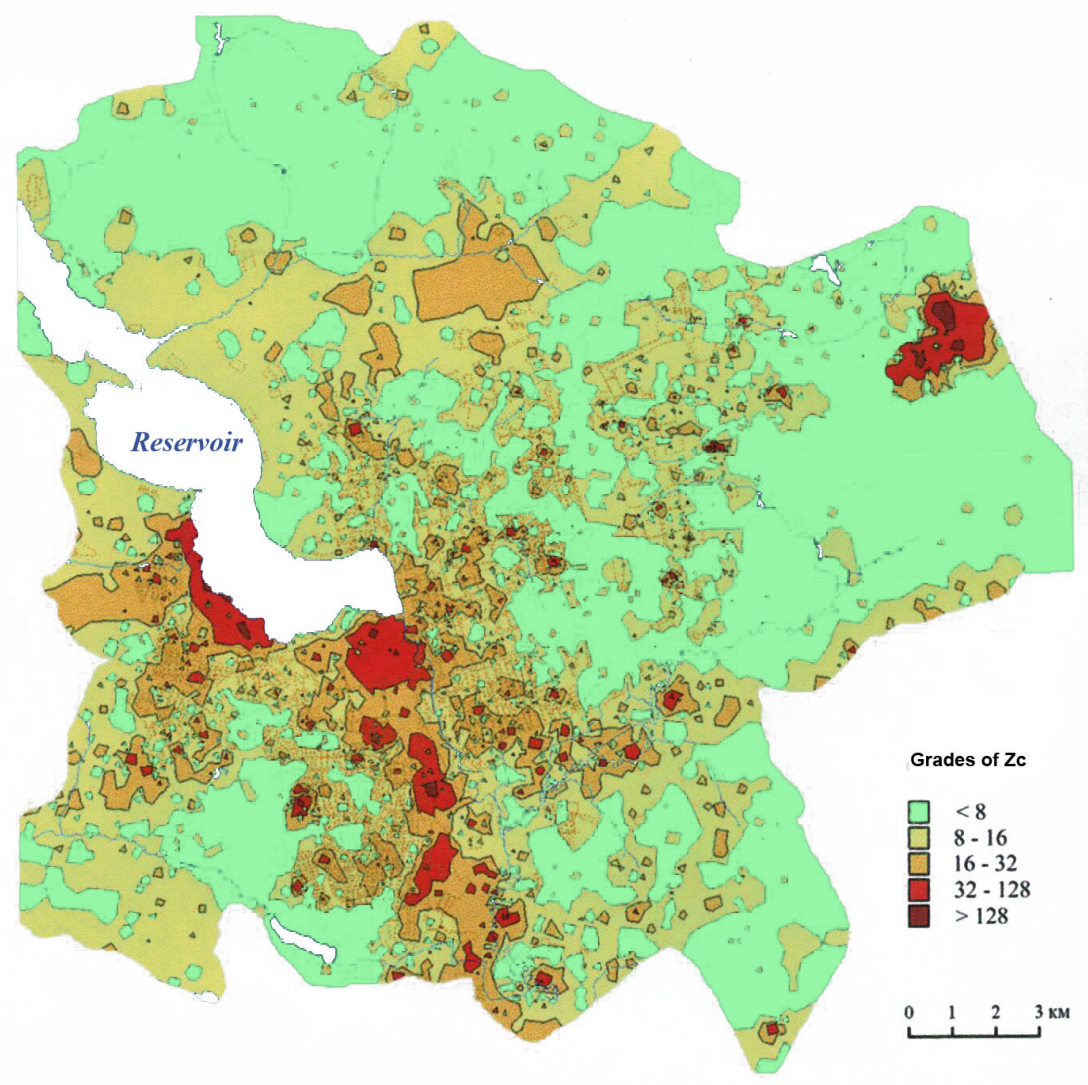

Figure 1: $\quad$ Map of total contamination indexes Zc.

slopes; $80 \%$ of the areas with permissible contamination $(\mathrm{Zc}<16)$ are located in the watersheds. These observations reflect unfortunate location of old industrial zones in the river valleys, where air flow is reduced, but pollutant deposition (so called "ion flow") is maximal.

Geochemical processes of element migration in urban landscapes are multiform and complex. Urban soils are usually more alkaline than their natural counterparts and tend to contain more organic matter. These features facilitate accumulation of cation-forming elements ( $\mathrm{Mn}, \mathrm{Cu}, \mathrm{Zn}, \mathrm{Pb}, \mathrm{Cd}, \mathrm{Ni}, \mathrm{Co}$, etc.) and migration of anion-forming elements (Cr, etc.) [5]. Therefore, excessive concentration of cation-forming elements is several times greater than these for anion-forming elements.

Soil contamination primarily depends on actual and former land use rather than on natural features of soil. Landscapes in Izhevsk can be classified as:

- natural (forests, bogged floodplains);

- partially transformed (man-made parks, gardens, kitchen-gardens, cemeteries);

- technogenic (residential, commercial, industrial areas; transport infrastructure). 


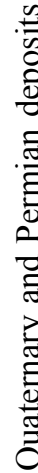

\begin{tabular}{|c|c|c|c|c|c|c|}
\hline \multicolumn{2}{|c|}{ 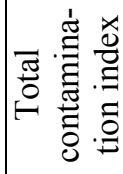 } & $\begin{array}{l}\widetilde{N} \\
\text { ปn }\end{array}$ & 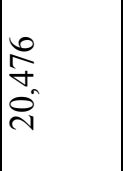 & 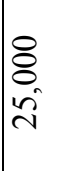 & $\begin{array}{l}\tilde{n} \\
\tilde{n} \\
\equiv\end{array}$ & 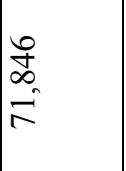 \\
\hline \multirow{8}{*}{ 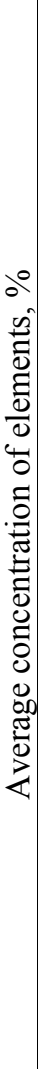 } & $\vec{F}$ & $\begin{array}{l}n \\
\\
\\
0 \\
0\end{array}$ & $\frac{n}{=}$ & $\begin{array}{l}n \\
\text { ñ } \\
\text { ñ }\end{array}$ & $\begin{array}{l}\dot{\infty} \\
\infty \\
m \\
\tilde{n} \\
0\end{array}$ & 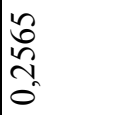 \\
\hline & Ü & $\begin{array}{l}\overrightarrow{0} \\
\text { Oे } \\
0 \\
0\end{array}$ & 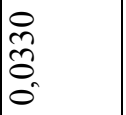 & $\begin{array}{l}\infty \\
\infty \\
\delta \\
\delta \\
0\end{array}$ & $\frac{\infty}{\infty}$ & $\begin{array}{l}0 \\
\infty \\
0 \\
2 \\
0\end{array}$ \\
\hline & 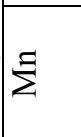 & $\frac{N}{N}$ & $\frac{n}{\stackrel{n}{c}}$ & $\mid \begin{array}{l}\infty \\
\pm \\
\pm \\
0\end{array}$ & $\underline{n}$ & 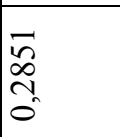 \\
\hline & ن & $\begin{array}{l}\text { ते } \\
\text { ठิ } \\
0 \\
0\end{array}$ & 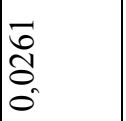 & $\begin{array}{l}\bar{n} \\
\delta \\
0 \\
0\end{array}$ & $\begin{array}{l}\frac{n}{8} \\
0 \\
0 \\
0\end{array}$ & $\frac{\pi}{0}$ \\
\hline & $\bar{z}$ & $\begin{array}{l}0 \\
\stackrel{2}{8} \\
0 \\
0\end{array}$ & 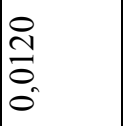 & $\begin{array}{l}0 \\
\overline{0} \\
0 \\
0\end{array}$ & \begin{tabular}{l}
0 \\
\multirow{2}{0}{} \\
0 \\
0 \\
0
\end{tabular} & 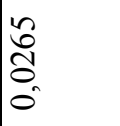 \\
\hline & ت & 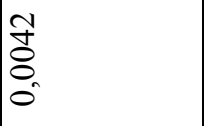 & \begin{tabular}{l}
0 \\
\multirow{2}{0}{} \\
8 \\
0 \\
0
\end{tabular} & $\begin{array}{l}0 \\
0 \\
8 \\
0 \\
0\end{array}$ & 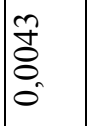 & o \\
\hline & Nี & $\begin{array}{l}0 \\
\equiv \\
0 \\
0 \\
0\end{array}$ & 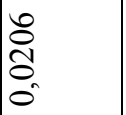 & $\begin{array}{l}\mathcal{N} \\
\delta \\
\delta \\
\delta\end{array}$ & $\begin{array}{l} \pm \\
0 \\
0 \\
0\end{array}$ & $\frac{\hat{a}}{0}$ \\
\hline & $\overrightarrow{2}$ & $\begin{array}{l}\infty \\
2 \\
2 \\
0 \\
0 \\
0\end{array}$ & $\begin{array}{l}\bar{g} \\
8 \\
0 \\
0\end{array}$ & 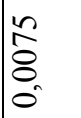 & $\frac{n}{n}$ & 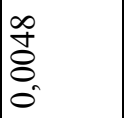 \\
\hline 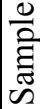 & 㭉 & ñ & $\stackrel{n}{\stackrel{g}{0}}$ & হે & 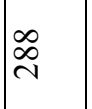 & $\stackrel{\infty}{\sim}$ \\
\hline \multicolumn{2}{|c|}{ 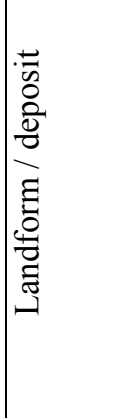 } & 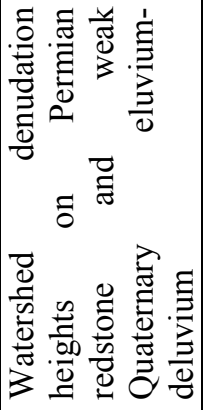 & 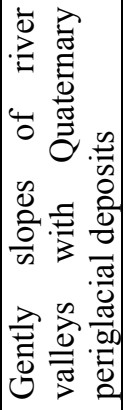 & 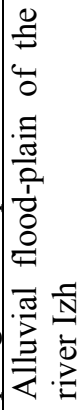 & 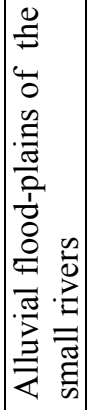 & 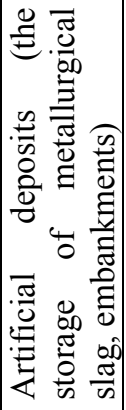 \\
\hline
\end{tabular}

WIT Transactions on Ecology and the Environment, Vol 141, (C) 2010 WIT Press www.witpress.com, ISSN 1743-3541 (on-line) 


\begin{tabular}{|c|c|c|c|c|c|c|c|c|c|c|c|c|c|c|c|c|c|c|c|}
\hline & 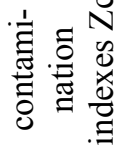 & 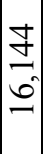 & $\begin{array}{l}0 \\
\infty \\
\infty \\
n \\
-1\end{array}$ & $\begin{array}{l}\Delta \\
\vec{\sigma} \\
\vec{\sim}\end{array}$ & $\begin{array}{l}0 \\
2 \\
0 \\
\pm \\
\pm\end{array}$ & $\begin{array}{l}7 \\
\infty \\
\infty \\
\infty \\
m\end{array}$ & $\begin{array}{l}n \\
n \\
n \\
n\end{array}$ & $\mid \begin{array}{c}\infty \\
0 \\
0 \\
n \\
-1\end{array}$ & $\begin{array}{l}= \\
\infty \\
\text { f }\end{array}$ & $\mid \begin{array}{l}n \\
0 \\
0 \\
0\end{array}$ & $\begin{array}{l}\stackrel{一}{ \pm} \\
\stackrel{5}{ \pm}\end{array}$ & $\begin{array}{c}\hat{\sigma} \\
\dot{f} \\
\hat{0} \\
0\end{array}$ & 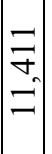 & $\begin{array}{c}2 \\
\delta \\
\infty \\
\vdots\end{array}$ & 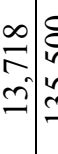 & 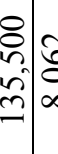 & 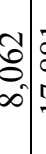 & $\begin{array}{l}\bar{\infty} \\
\infty \\
\sim \\
=\end{array}$ & $\underset{+}{\stackrel{+}{+}}$ \\
\hline & $\ddot{E}$ & $\begin{array}{l}1 \\
6 \\
\infty \\
1 \\
0 \\
0\end{array}$ & 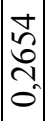 & 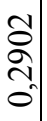 & $\begin{array}{l}n \\
\stackrel{n}{ } \\
m \\
n \\
0\end{array}$ & 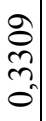 & $\begin{array}{l}\infty \\
n \\
n \\
\\
0\end{array}$ & $\begin{array}{c}w \\
\tilde{2} \\
\tilde{2} \\
0\end{array}$ & $\begin{array}{l}\hat{n} \\
\infty \\
\text { vै } \\
0\end{array}$ & $\mid \begin{array}{c}m \\
\hat{2} \\
\tilde{a} \\
0\end{array}$ & $\begin{array}{l}2 \\
8 \\
n \\
n \\
0\end{array}$ & $\left|\begin{array}{c}0 \\
0 \\
0 \\
0 \\
0\end{array}\right|$ & $\mid \begin{array}{c}m \\
n \\
\tilde{n} \\
0\end{array}$ & 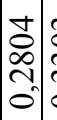 & 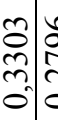 & \begin{tabular}{l|l}
0 & 1 \\
2 & \multicolumn{2}{c}{} \\
& $\alpha$ \\
0 & 0
\end{tabular} & 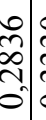 & 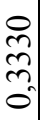 & $\begin{array}{l}a \\
\frac{n}{n} \\
0\end{array}$ \\
\hline & $\dot{U}$ & $\begin{array}{l}\infty \\
\bar{d} \\
0 \\
0\end{array}$ & 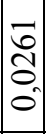 & $\begin{array}{c}0 \\
\widetilde{2} \\
\hat{\delta} \\
0 \\
0\end{array}$ & $\begin{array}{l}5 \\
2 \\
0 \\
0 \\
0\end{array}$ & 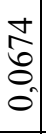 & \begin{tabular}{l}
\multirow{0}{0}{} \\
0 \\
0 \\
0
\end{tabular} & 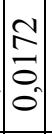 & 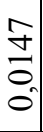 & $\mid \begin{array}{c}m \\
m \\
0 \\
0\end{array}$ & $\begin{array}{l}\text { Nิ } \\
\text { Oे } \\
0 \\
0\end{array}$ & $\left|\begin{array}{l}2 \\
\sigma \\
0 \\
0\end{array}\right|$ & $\mid \begin{array}{l}0 \\
2 \\
0 \\
0\end{array}$ & 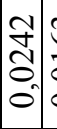 & 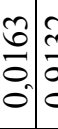 & \begin{tabular}{l|l}
2 & \multicolumn{2}{c}{} \\
$\tilde{\sigma}$ & $\bar{c}$ \\
0 & $c$
\end{tabular} & 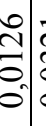 & 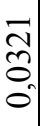 & $\begin{array}{l}a \\
\frac{n}{0} \\
0\end{array}$ \\
\hline 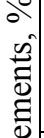 & $\Xi$ & \begin{tabular}{l}
2 \\
$\hat{n}$ \\
\hdashline \\
0
\end{tabular} & 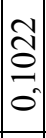 & $\begin{array}{c} \pm \\
8 \\
0 \\
0 \\
0\end{array}$ & 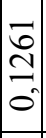 & $\begin{array}{c}N \\
\frac{N}{0} \\
0\end{array}$ & 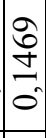 & $\left|\begin{array}{l}n \\
0 \\
8 \\
0 \\
0\end{array}\right|$ & $\begin{array}{l}\infty \\
\sigma \\
0 \\
0\end{array}$ & $\left|\begin{array}{c}0 \\
\infty \\
0 \\
0 \\
0\end{array}\right|$ & $\begin{array}{l}\stackrel{a}{n} \\
\stackrel{2}{0} \\
0\end{array}$ & 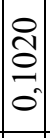 & $\left|\begin{array}{c}0 \\
0 \\
\vdots \\
0 \\
0\end{array}\right|$ & 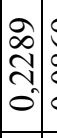 & \begin{tabular}{l|l}
0 & $\mid r$ \\
0 & \\
$\infty$ & $=$ \\
0 & $=$ \\
0 & $c$
\end{tabular} & 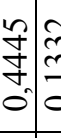 & \begin{tabular}{l|l} 
& \\
2 & \\
$m$ & \\
0 &
\end{tabular} & $\begin{array}{l}0 \\
i n \\
\\
0\end{array}$ & $\begin{array}{l}\text { オे } \\
\infty \\
0 \\
0 \\
0\end{array}$ \\
\hline $\begin{array}{l}\overline{0} \\
4 \\
0 \\
0 \\
0 \\
.0\end{array}$ & ن & $\begin{array}{l}m \\
0 \\
0 \\
0\end{array}$ & \begin{tabular}{l} 
\pm \\
\hdashline \\
0 \\
0
\end{tabular} & $\begin{array}{l}0 \\
\frac{6}{8} \\
0 \\
0\end{array}$ & $\begin{array}{l}n \\
\vdots \\
8 \\
0 \\
0\end{array}$ & 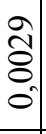 & $\mid$\begin{tabular}{l}
$\infty$ \\
\hdashline \\
0 \\
0
\end{tabular} & $\begin{array}{l}n \\
0 \\
8 \\
0 \\
0\end{array}$ & $\begin{array}{l}\stackrel{\circ}{8} \\
8 \\
\circ \\
\circ\end{array}$ & 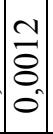 & $\frac{\infty}{0}$ & $\mid$\begin{tabular}{c}
$m$ \\
\hdashline \\
0 \\
0 \\
0
\end{tabular} & 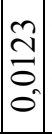 & $\left|\begin{array}{c}0 \\
\hdashline \\
0 \\
0 \\
0\end{array}\right|$ & \begin{tabular}{l|l}
$n$ \\
\\
0 \\
0
\end{tabular} & 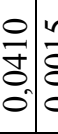 & $\begin{array}{l}n \\
8 \\
0 \\
0 \\
0\end{array}$ & $\begin{array}{l}\searrow \\
\\
0 \\
0\end{array}$ & $\begin{array}{l}\stackrel{\sigma}{0} \\
0 \\
0\end{array}$ \\
\hline 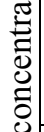 & $\bar{z}$ & 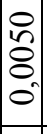 & 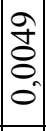 & $\left|\begin{array}{l}3 \\
0 \\
8 \\
0 \\
0\end{array}\right|$ & $\begin{array}{l}1 \\
0 \\
8 \\
0 \\
0\end{array}$ & $\begin{array}{c}n \\
\frac{n}{6} \\
0\end{array}$ & $\begin{array}{l}\text { 응 } \\
8 \\
8 \\
0\end{array}$ & $\left|\begin{array}{l}6 \\
0 \\
8 \\
0 \\
0\end{array}\right|$ & $\mid$\begin{tabular}{l}
$n$ \\
\multirow{8}{*}{} \\
8 \\
0 \\
0
\end{tabular} & $\mid \begin{array}{l}n \\
\stackrel{n}{0} \\
8 \\
0\end{array}$ & $\begin{array}{l}8 \\
0 \\
8 \\
0 \\
0\end{array}$ & $\mid \begin{array}{l}\hat{y} \\
8 \\
0 \\
0\end{array}$ & $\mid \begin{array}{c}\bar{n} \\
8 \\
0 \\
0\end{array}$ & \begin{tabular}{l}
0 \\
\multirow{\Xi}{0}{} \\
8 \\
0
\end{tabular} & 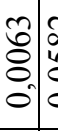 & 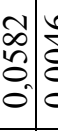 & $\begin{array}{l}0 \\
8 \\
8 \\
0 \\
0\end{array}$ & $\begin{array}{l}\text { ㅇ } \\
8 \\
8 \\
0\end{array}$ & 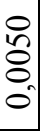 \\
\hline 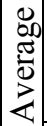 & ت & 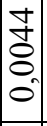 & 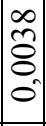 & \begin{tabular}{l}
$n$ \\
\multirow{2}{0}{} \\
0 \\
0 \\
0
\end{tabular} & $\mid \begin{array}{l}0 \\
\infty \\
\delta \\
0 \\
0 \\
0\end{array}$ & $\begin{array}{l}\overline{6} \\
8 \\
0 \\
0 \\
0\end{array}$ & 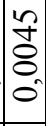 & 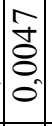 & $\left|\begin{array}{l}n \\
\multirow{2}{0}{} \\
0 \\
0 \\
0\end{array}\right|$ & $\left|\begin{array}{l}n \\
\tilde{\delta} \\
0 \\
0 \\
0\end{array}\right|$ & 奋 & $\left|\begin{array}{l}0 \\
\stackrel{0}{8} \\
0 \\
0\end{array}\right|$ & $\left|\begin{array}{l}\tilde{n} \\
\tilde{8} \\
0 \\
0\end{array}\right|$ & 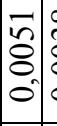 & 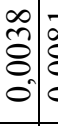 & $\begin{array}{l}\infty \\
\infty \\
\delta \\
0 \\
0\end{array}$ & 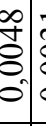 & $\begin{array}{l}\bar{\delta} \\
\delta \\
0 \\
0\end{array}$ & 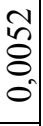 \\
\hline & Nี & $\begin{array}{l}\stackrel{m}{f} \\
\stackrel{0}{0} \\
0\end{array}$ & $\mid \begin{array}{l}\infty \\
n \\
0 \\
0 \\
0\end{array}$ & 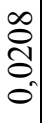 & $\begin{array}{c}N \\
\vdots \\
0 \\
0\end{array}$ & 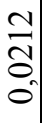 & $\begin{array}{l}7 \\
0 \\
0\end{array}$ & $\begin{array}{l}\exists \\
\bar{\sigma} \\
0 \\
0\end{array}$ & $\left|\begin{array}{c}m \\
\infty \\
0 \\
0 \\
0 \\
0\end{array}\right|$ & $\left|\begin{array}{l}0 \\
\infty \\
8 \\
0 \\
0\end{array}\right|$ & $\begin{array}{l} \pm \\
\\
0 \\
0\end{array}$ & $\left|\begin{array}{c}\tilde{N} \\
\tilde{\sigma} \\
0 \\
0\end{array}\right|$ & $\mid \begin{array}{l} \pm \\
0 \\
0 \\
0 \\
0\end{array}$ & $\begin{array}{l}n \\
\infty \\
0 \\
0 \\
0\end{array}$ & 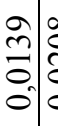 & 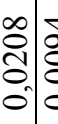 & 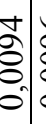 & $\begin{array}{l}0 \\
\circ \\
8 \\
\circ \\
0\end{array}$ & $\mid \begin{array}{c}0 \\
0 \\
0 \\
0 \\
0 \\
0\end{array}$ \\
\hline & $\hat{2}$ & $\mid \begin{array}{l}0 \\
0 \\
0 \\
8 \\
0 \\
0 \\
\mid\end{array}$ & \begin{tabular}{l}
$\infty$ \\
\hdashline \\
$\varnothing$ \\
$\varnothing$ \\
0 \\
0
\end{tabular} & $\begin{array}{l}n \\
\frac{n}{8} \\
0 \\
0\end{array}$ & 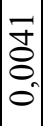 & 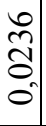 & 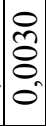 & $\mid$\begin{tabular}{l}
$n$ \\
\multirow{8}{0}{} \\
8 \\
0
\end{tabular} & $\mid$\begin{tabular}{l}
$\infty$ \\
\hdashline \\
$\delta$ \\
0
\end{tabular} & $\left|\begin{array}{c}\infty \\
0 \\
0 \\
0 \\
0\end{array}\right|$ & $\begin{array}{l}0 \\
\text { ஜn } \\
8 \\
0\end{array}$ & $\left|\begin{array}{l}\hat{\infty} \\
0 \\
0 \\
0 \\
0\end{array}\right|$ & 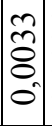 & 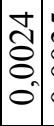 & 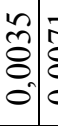 & $\begin{array}{l}\bar{\delta} \\
\delta \\
\delta\end{array}$ & 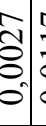 & $\begin{array}{l}\bar{Z} \\
\bar{\Xi} \\
0\end{array}$ & 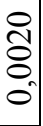 \\
\hline & 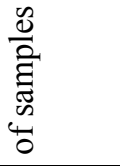 & 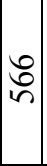 & $\begin{array}{l}\infty \\
n\end{array}$ & 寸 & $\begin{array}{l}0 \\
\text { } \\
\text { n. }\end{array}$ & $\begin{array}{l}\mathbb{J} \\
\text { N }\end{array}$ & $\stackrel{q}{+}$ & $\overline{\mathrm{\sigma}}$ & $\stackrel{\mathscr{P}}{\sim}$ & ڤి & $\begin{array}{l}n \\
n \\
n\end{array}$ & ○ & 寻 & O & શે= & $=1$ & $=$ & ㄱ & $\stackrel{\oplus}{\oplus}$ \\
\hline 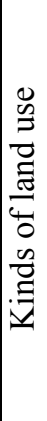 & & 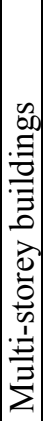 & 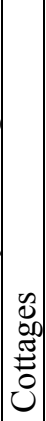 & 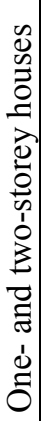 & 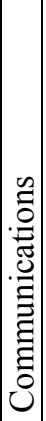 & 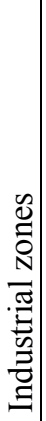 & 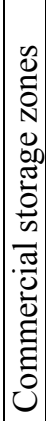 & 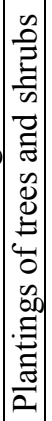 & $\begin{array}{l}n \\
\frac{n}{3} \\
\tilde{z} \\
\tilde{n}\end{array}$ & 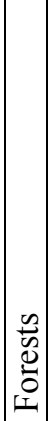 & 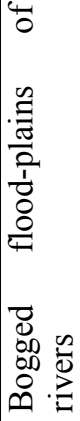 & 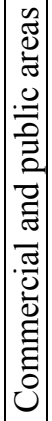 & 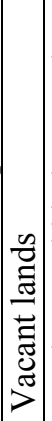 & 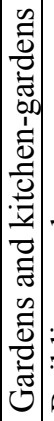 & 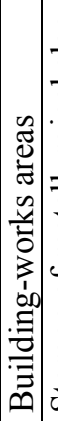 & 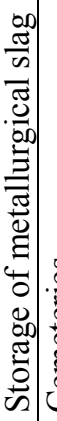 & 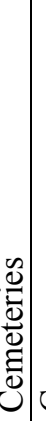 & 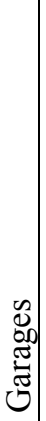 & 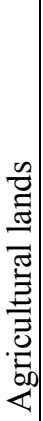 \\
\hline
\end{tabular}


Table 2 presents average concentration of elements and total contamination indexes $\mathrm{Zc}$ in different types of landscape.

As the tables show, grade of contamination strongly depends on actual land use, but also on former land use and migration of pollutants. For example, several anomaly cases in modern low-rise residential and trees/shrubs zones are caused by previous use of these lands for slag deposition and nearby location of industry parks.

Urban environment includes numerous colored dyed metal objects such as cars or roofs. All of them take part in soil contamination because of corrosion processes. Background level of contamination $(\mathrm{Zc} \approx 8-9)$ is detected in gardens and kitchen-gardens, cemeteries, agricultural lands. Industry and waste pollution superimpose over these values.

\section{Clean-up of pesticide contamination in a city district}

One of the residential districts in Izhevsk was built in the 1970-80s on the site of disused airport. The developing organization neglected to utilize the reminder of pesticides for agricultural aviation (DDT and GHCG). Instead, the hazardous chemicals were buried in the nearest ravine by a bulldozer. Several multi-storey apartment buildings and a nursery school were built close to the burial place. The concentration of contaminants was so high, that the inhabitants could literally smell it, especially after rains.

The City Committee of Environment Protection carried out a detailed site investigation and revealed pesticide concentration up to $50 \mathrm{mg} / \mathrm{kg}$ (500 times more than permissible level). Permissible level of DDT and GHCG in soils established in Russia 0,1 mg/kg; in Germany (Berlin and Brandenburg) 0,5 $\mathrm{mg} / \mathrm{kg}$; in The Netherlands (agricultural lands) $0,0025 \mathrm{mg} / \mathrm{kg}$ [2].

Water streams in the downslope channel spread pesticides along the ravine, raising pesticide concentration in the bottom deposits up to 10 times excess of Russian maximum permissible norm.

The clean-up project was completed later in the 1990s by excavation of contaminated soil. No significant human health impact was detected according to the medical statistics. A new multi-storey building was constructed on the remediated site several years after.

\section{Cleanup of the former plant site from lubricating oil}

Izhevsk ball bearing plant was built in the 1950s on the bank of the small river Karlutka, on its slope and floodplain. This area was not the best choice for industrial engineering because of ground water activity and slope processes. The plant's territory was located too close to the river channel, what is inadmissible under the terms of current legislation.

The plant required 70 tons of oil a year for lubrication of lathe machines. Oil circulation process was unreliable, causing an increasing soil contamination. Lubricating oil was kept in underground tanks which suffered from corrosion 
and leaked badly. Another source of contamination was service water used for washing metal shavings off the machines.

Severe oil contamination of the river Karlutka (up to $6,5 \mathrm{mg} / \mathrm{dm}^{3}, 130$ times more than permissible level) was discovered in the 1990s by environmental investigations. Several oil springs were found in the lower part of the river slope, near the Karlutka. An attempt to protect the river using a clay screen had no effect.

At the same time, the plant started to experience economic problems. The bankruptcy and several resells took place after the crisis of 1998. Eventually, the new owner decided to reconstruct the property as an office centre. As the fact of contamination was already known, the Ministry of Natural Resources demanded detailed investigation and rehabilitation of the plant site.

According to the Code of Practice SP 11-102-97 [2], 8 boreholes were drilled and 60 samples of soil and ground water were obtained. Based on this material, ecologists estimated quantity and distribution of the contaminant and made a $3 \mathrm{D}$ model of the affected area. The quantity was of 70 tons of lubricating oil and $10500 \mathrm{~m}^{3}$ of contaminated soil; the total depth of contamination was about 8 meters.

The remediation project involved excavation of contaminated soil where possible and drilling 5 bore-wells to pump out the oil. The wells of $800 \mathrm{~mm}$ in diameter and 5-7 $\mathrm{m}$ depth are located in the most polluted points and/or lower parts of relief. The pumping started in 2005 and still goes on. The amounts of pumped oil started from $1 \mathrm{t}$ per month in 2005 and has considerably decreased by now, which signifies near completion.

\section{The project of an artificial geochemical barrier for protection of the city reservoir}

Metallurgical slag had been depositing into the cove of the Izhevsk reservoir since 1760 till the $1980 \mathrm{~s}$. The storage area spans $1,2 \mathrm{~km}^{2}$ with the average thickness of 5 meters, while the depth of ground water is 1-1,5 m. A significant amount of slag was dumped directly into the water and therefore lay beyond the reservoir level. All of this slag is still contacting with the city's main water source.

A group of ecologists lead by me explored the slag storage in 2006 and revealed numerous pools filled with alkaline water $(\mathrm{pH} 10-11)$. Such water is unnaturally bluish and lifeless. The subsurface water can be even more polluted, but the hard structure of slag leaves no possibility to examine it by drilling or digging.

Alkalinity of the water is formed by alkaline components of slag, such as $\mathrm{CaO}$ (up to $56 \%$ of slag) and $\mathrm{MgO}$ (up to $20 \%$ of slag). Alkaline water forms suitable environment for migration of 6-valent chromium, one of the toxic steel components. Concentration of 6-valent chromium in surface water of the slag storage goes from 0,006 to $0,677 \mathrm{dm}^{3}$, up to 34 times more than permissible norm. Fortunately, the water doesn't transfer other elements such us iron or manganese. 
As mentioned above, traditional remediation approach is not applicable for the slag storage. Since excavation is not possible, the project of reservoir protection is based on creation of an artificial geochemical barrier for chromium ion deposition. Construction materials for the barrier should be accessible and cheap.

A series of laboratory experiments has been set in order to select the material. According to these studies, the natural peat was chosen as an absorption agent for chromium ions. The peat did a good job in decreasing chromium concentration; however it turned out to force the lixiviation of iron, manganese and organic substances. It was decided to use limestone in order to compensate these effects.

The results of experiments are presented in the table 3 .

Table 3: Characteristics of water before and after filtration through peat and limestone.

\begin{tabular}{|l|c|c|c|c|c|}
\hline \multirow{2}{*}{ Conditions of experiment } & \multicolumn{4}{|c|}{ Characteristics of water } \\
\cline { 2 - 6 } & $\mathrm{pH}$ & $\begin{array}{c}\mathrm{Fe}, \\
\mathrm{Mg} / \mathrm{dm}^{3}\end{array}$ & $\begin{array}{c}\mathrm{Mn}, \\
\mathrm{Mg}^{2} \mathrm{dm}^{3}\end{array}$ & $\begin{array}{c}\mathrm{Cr}^{3+}, \\
\mathrm{Mg} / \mathrm{dm}^{3}\end{array}$ & $\begin{array}{c}\mathrm{Cr}^{6+}, \\
\mathrm{Mg}^{6} \mathrm{dm}^{3}\end{array}$ \\
\hline Before filtration & 9,78 & 0,12 & 0,015 & $<0,01$ & 0,022 \\
\hline After filtration through peat & 6,49 & 0,39 & 0,03 & 0,01 \\
\hline $\begin{array}{l}\text { After filtration through peat } \\
\text { and limestone }\end{array}$ & 7,39 & 0,136 & 0,029 & \multicolumn{2}{|c|}{0,001} \\
\hline
\end{tabular}

As a result, our group suggested protecting Izhevsk reservoir by a complex geochemical barrier of 3 vertical layers (Fig. 2). The first layer of peat is intended to create conditions for acid anaerobic reduction. These conditions facilitate transformation of toxic 6-valent chromium into less toxic 3-valent chromium. The following spontaneous reaction occurs in acid conditions:

$$
\mathrm{Cr}^{6+}+\operatorname{Rorg} \stackrel{3 \mathrm{H}^{+}}{\longrightarrow} \mathrm{Cr}^{3+}
$$

Rorg - organic reductant

The intermediate layer of sand and gravel is meant to separate peat and limestone from each other. The third layer of limestone provides alkaline conditions in order to deposit $\mathrm{Fe}^{2+}, \mathrm{Mn}^{2+}$ and to neutralize organic acids of peat.

$$
\begin{gathered}
2 \mathrm{FeO}+\mathrm{O}_{2} \longrightarrow \mathrm{Fe}_{2} \mathrm{O}_{3} \downarrow \\
2 \mathrm{Mn}(\mathrm{OH})_{2}+2 \mathrm{H}_{2} \mathrm{O}+\mathrm{O}_{2} \longrightarrow 2 \mathrm{Mn}(\mathrm{OH})_{4} \downarrow
\end{gathered}
$$



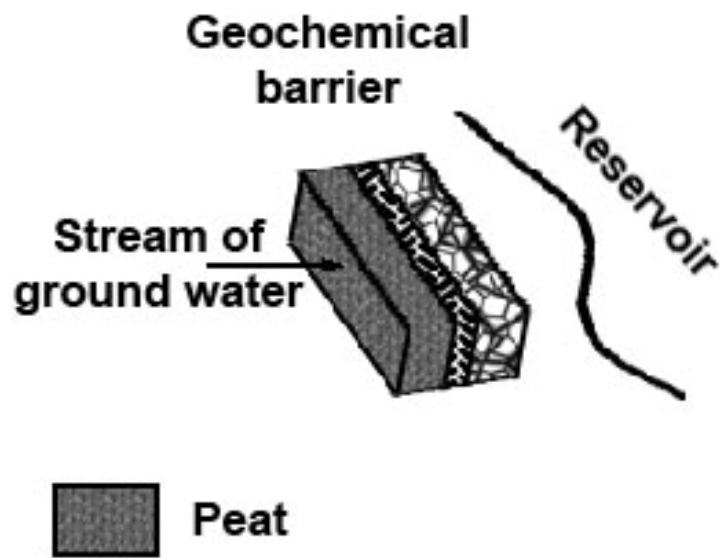

\section{Sand and gravel}

\section{2imestone}

Figure 2: $\quad$ The scheme of geochemical barrier.

The project of geochemical barrier is a part of the city's reservoir reconstruction program. Other measures include removal of contaminated ground silt, filling up bogged shallows and renovation of the banks. The barrier is planned to be constructed in a trench under the new embankment.

\section{Conclusion}

Contaminated lands are very common in industrial cities of Russia. Discrepancy between environmental and economic attitudes to the problem holds detains rehabilitation of brownfields.

The major government's contribution was subsidizing of geochemical mapping procedure, which was carried out in many cities and formed a solid foundation for future investigations. The mapping results are suitable for revealing and quantitative investigation of soil contamination within the whole city. On the other side, there is no any federal, regional or even municipal system for registration of contaminated or degraded lands.

Legislation makes the land owners responsible both for prevention and rehabilitation of degraded lands (brownfields). Obviously, such a broad rule does not always work well. Identification of polluters and gaining compensation is often impossible, especially in case of contamination within old industrial zones. So, in fact all expenditures concerning contaminated and degraded lands charge new owners. It means additional risk for investors. 
As was shown above, the situation with brownfields in Izhevsk is controversial and has much potential for improvement. Therefore European and American experience in the sphere of brownfields rehabilitation is of great importance.

\section{References}

[1] Turning brownfields into greenbacks: developing and financing environmentally contaminated urban real estate / Robert A. Simons. Washington, D.C.: Urban Land Institute, 1998. HD257 .S55 1998.

[2] Engineering environmental site investigations for construction. Code of practice. SP 11-102-97. Moscow, 1997. (Published in Russian: Свод правил. СП 11-102-97 «Инженерно-экологические изыскания для строительства». М.: Госстрой России, 1997. 41 с.)

[3] Environmental Geochemistry - Moscow, 1990. (Published in Russian: Геохимия окружающей среды./ Сает Ю.А., Ревич Б.А., Янин Е.П. "Недра", Москва, 1990, 335 с.)

[4] Sanka M., Strnad M., Vondra J., Paterson E. 1995. Sources of soil and plant contamination in an urban environment and possible assessment methods. Intern. J. Environ.Anal.Chem., 59:327-343.

[5] Ecogeochemistry of urban landscapes / Editor-in-chief N.S. Kasimov. Moscow, 1995. (Published in Russian: Экогеохимия городских ландшафтов / Под ред. Н.С. Касимова. М.: Изд. МГУ, 1995. 336 с.) 